Meta

Journal des traducteurs

Translators' Journal

\title{
Anthroponymes en textes de théâtre : drôles de noms propres
}

\section{Thierry Gallèpe}

Volume 51, numéro 4, décembre 2006

La traduction des noms propres (1) et Langue, traduction et mondialisation : interactions d'hier, interactions d'aujourd'hui Language, Translation and Globalization: Interactions from Yesterday, Interactions from Today (2)

URI : https://id.erudit.org/iderudit/014332ar

DOI : https://doi.org/10.7202/014332ar

Aller au sommaire du numéro

Éditeur(s)

Les Presses de l'Université de Montréal

ISSN

0026-0452 (imprimé)

1492-1421 (numérique)

Découvrir la revue

Citer cet article

Gallèpe, T. (2006). Anthroponymes en textes de théâtre : drôles de noms propres. Meta, 51(4), 651-659. https://doi.org/10.7202/014332ar

\section{Résumé de l'article}

Les textes théâtraux sont particulièrement adaptés à l'identification formelle des noms propres dans la mesure où ils apparaissent à deux endroits bien définis : les listes composant le paratexte et les didascalies qui font partie du texte. Cette contribution est dédiée en grande partie aux caractéristiques sémantiques des noms propres en regard des problèmes de traduction. Il est ainsi possible d'identifier trois sortes de composantes sémantiques ressortant du domaine de la connotation. Celles-ci déterminent à la fois la structure de signification et expliquent les difficultés de traduction.
Ce document est protégé par la loi sur le droit d'auteur. L'utilisation des services d’Érudit (y compris la reproduction) est assujettie à sa politique d'utilisation que vous pouvez consulter en ligne.

https://apropos.erudit.org/fr/usagers/politique-dutilisation/ 


\title{
Anthroponymes en textes de théâtre: drôles de noms propres
}

\author{
THIERRY GALL ì PE \\ Université de Tours, Tours, France \\ thierry.gallepe@univ-tours.fr
}

\begin{abstract}
RÉSUMÉ
Les textes théâtraux sont particulièrement adaptés à l'identification formelle des noms propres dans la mesure où ils apparaissent à deux endroits bien définis: les listes composant le paratexte et les didascalies qui font partie du texte. Cette contribution est dédiée en grande partie aux caractéristiques sémantiques des noms propres en regard des problèmes de traduction. Il est ainsi possible d'identifier trois sortes de composantes sémantiques ressortant du domaine de la connotation. Celles-ci déterminent à la fois la structure de signification et expliquent les difficultés de traduction.
\end{abstract}

\begin{abstract}
Theater texts are particularly suited to formal identification of proper names since they appear in two given places, the first one belonging to the paratext, the lists, and the second, called "name identifications," being part of the text itself. This paper, however, is mainly dedicated to the semantic characteristics of these proper names, with regards to translation problems. It is thus possible to identify three types of semantic features belonging to the domain of connotations that both determine their meaning structure and explain their translation difficulties.
\end{abstract}

\section{MOTS-CLÉS/KEYWORDS}

nom propre, traduction, paratexte, didascalies, connotation

Le but poursuivi dans cet article est d'observer les noms propres, et plus spécifiquement les anthroponymes, dans les textes de théâtre afin de dégager quelques traits pertinents pour leur description, avec, de surcroît, les conséquences que cela peut avoir sur leur traduction.

Le travail mené à cette fin s'est essentiellement accompli à partir d'un corpus de textes dramatiques allemands. Mais quelques textes français ont été aussi mis à contribution!

\section{Critères formels en texte de théâtre}

Je ne désire pas m’appesantir longuement sur cette dimension. Les pièces de théâtre assignent aux anthroponymes un lieu privilégié, celui du baptême, en quelque sorte: il s'agit de ce que j'appelle (Gallèpe 1997a) la liste, composante du paratexte théâtral (avec les titres et les bornes). C'est ici que les personnages sont présentés (le plus souvent ${ }^{1}$ ). Formellement, les anthroponymes se trouvent donc à gauche de la flèche ou des deux-points, ou seuls sous forme de liste en colonne ou en ligne.

La difficulté est que ces listes comportent aussi des indications non anthroponymiques, telles que "Zeitgenossen aller Art» (Zuckmayer; Der Hauptmann von Köpenick), "andere Personen, fruchtbar und furchtbar» (Kroetz; Bauerntheater), etc. 
La même difficulté se retrouve avec l'autre endroit stratégique, où se trouvent dans les textes dramatiques les anthroponymes: les didascalies en marge gauche, qui servent de point d'ancrage aux répliques tenues par les personnages, et que je nomme les Indications de Nom (IdN) (Gallèpe, 1997a).

Les autres critères rejoignent ceux plus classiques concernant la graphie, la présence de déterminatif et les complexes nominaux (bases nominales complexes) tels que «Graf Fontana» ou «Oberst Serge» (Hochhuth; Der Stellvertreter). Ces traits étant non spécifiques des textes de théâtre, je ne m'y attacherai pas plus avant, même s'il convient de noter une spécificité formelle de ce genre de discours: il s'agit de l'absence du marqueur d'identification lorsque la description définie (ou indéfinie ou non définie) des listes fonctionne comme IdN:

\begin{tabular}{|l|l|l|}
\hline Liste & IdN & Pièce \\
\hline Maria Schmitt, seine Mutter & MUTTER & Bauerntheater \\
\hline Der Minister a. D. & MINISTER & Inselkomödie \\
\hline Ein Diener bei Bernhardi & DIENER & Professor Bernhardi \\
\hline
\end{tabular}

\section{Caractéristique spécifique des anthroponymes théâtraux}

Il convient de rappeler avant tout le dispositif énonciatif particulier des textes de théâtre comme signe, c'est-à-dire leur fonctionnement à double détente diégétique:

- Le premier niveau de construction mentale se rapporte à la constitution d'une diégèse (que l'on peut qualifier de scénique) à partir de la lecture du texte (qui permet éventuellement de construire une représentation scénique concrète, quand le lecteur est le metteur en scène).

- Le second niveau est la construction d'une autre diégèse (la vraie?) à partir de la représentation première. Elle reste signifiée mentalement, à partir du signifiant qu'est la diégèse scénique, que celle-ci soit seulement mentale ou au contraire réalisée sur scène.

Cette distinction est un critère utile pour la description des didascalies, notamment en ce qui concerne leur assise diégétique: sont-elles diégétiques (= de niveau 2) ou extra-diégétiques (de niveau 2) et donc (seulement) scéniques?

Ce dispositif particulier a quelques implications pour les opérations de référence construites avec les anthroponymes; en effet, si, le plus souvent, la deuxième diégèse reste unique, il y a possibilité de multiplication des diégèses scéniques (de niveau 1).

Arrivé à ce point, il faut rappeler une différence entre noms communs et noms propres: la pluralité référentielle est essentielle aux premiers, accidentelle aux seconds. Mais dans les cas de textes théâtraux, la pluralité a tendance à devenir moins accidentelle. Que l'on veuille bien considérer les exemples suivants:

«Je me rappelle les Monsieur Jourdain de mon enfance.»

«De tous les Tartuffe(s?) que j'ai vus, c'est le Tartuffe de Vitez que je préfère», etc.

On le voit donc ici, en liaison avec le dispositif sémiotique particulier inhérent aux textes de théâtre, un premier élément se doit être noté, qui semble rapprocher d'une certaine façon les noms propres des noms communs. 


\section{Les anthroponymes ont-il du sens?}

Le problème n'est pas anodin. Car de façon définitoire c'est l'absence de sens qui fonde d'une certaine manière la catégorie des noms propres. Ceux-ci ont en effet une référence, mais pas de sens. S. Mill précise qu'ils ont ainsi une dénotation (référent), mais pas de connotation (sens) ${ }^{2}$.

Cela rejoint donc la perspective de Kripke pour qui les noms propres ont un référent sans avoir de signification à proprement parler, et doivent désigner le même individu dans tous les mondes possibles, ce qui explique le concept de désignateur rigide qu'on peut leur appliquer.

Pourtant, selon Frege, aucune référence n'est possible sans sens. De quel sens peut-il s'agir alors? Pour répondre, il convient de faire la différence entre:

- la signification vériconditionnelle, qui correspond à la signification lexicale, permettant l'autonomie référentielle, qui se conçoit comme une signification descriptive;

- et la signification non vériconditionnelle, correspondant à une signification procédurale. C'est-à-dire qu'un ensemble d'instructions, la procédure, est attaché au "mot», à l'instar des indexicaux; ils n'ont donc pas d'autonomie référentielle; en d'autre termes, pas de signification lexicale qui permet d'effectuer l'opération de référence en autonomie: c'est-à-dire que l'on ne peut leur attribuer de référence actuelle sur la base de leur référence virtuelle, car ils n'en ont pas. Si les significations procédurales sont lexicalement attachées à des mots, il y a donc bien encodage linguistique, mais il n'y a pas à proprement parler de signification lexicale.

Il apparaît donc que si signification portée par les noms propres il y a, elle est sans aucun doute plutôt procédurale; telle est la position couramment adoptée. On ne saurait mettre en doute la dimension procédurale dans le fonctionnement des noms propres. Mais peut-on se satisfaire d'une telle position, qui exclut toute signification d'ordre lexical, sémantique?

Pour répondre à cette question, je dois me tourner vers la sémantique lexicale, afin d'examiner de plus près ce concept de signification.

Dans la théorie, plusieurs approches sont possibles. La plus courante est sans doute celle mettant à contribution l'opposition dénotation $\neq$ connotation, pour désigner les différentes couches de signification, composantes de la signification globale d'un terme.

Il serait certes plus satisfaisant de recourir à une description en termes de sèmes (inhérents $\neq$ afférents, à la F. Rastier (1996) tant il est vrai que le couple dénotation $\neq$ connotation ne brille pas toujours par sa grande rigueur. Mais dans le cadre de cet article, ces deux concepts, largement répandus, permettent de mener la démarche à

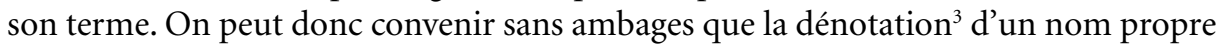
n'existe pas. Mais qu'en est-il des traits connotatifs?

Il apparait sans nul doute possible que les noms propres en sont bien porteurs. C'est du moins l'hypothèse que je vais étayer par l'étude qui suit.

\subsection{Connotation d'ordre énonciatif $=>$ situative de diégèse: localisation dans le temps et l'espace}

Les premières connotations étudiées permettent de mieux situer la diégèse dans le temps et l'espace dans la mesure où elles rendent possible une meilleure identification 
des locuteurs. Voilà pourquoi elles se situent dans l'ordre des connotations énonciatives (Kerbrat-Orecchioni 1977). Quelques exemples:

(1) Pierpont Mauler / Criddle / Lennox / Graham / Slift etc. (Brecht; Die heilige Johanna der Schlachthöfe);

(2) Georg Danton / Legendre / Camille Desmoulins / Hérault-Séchelles etc. (Büchner; Dantons Tod);

(3) Marquis de Sade / Jean Paul Marat / Simone Evrard / Charlotte Corday (Weiss; Die Verfolgung und Ermordung Jean Paul Marats dargestellt durch die Schauspielergruppe des Hospizes zu Charenton unter Anleitung des Herrn de Sade).

On voit bien que, par effet cumulatif de masse, une scène diégétique se dessine, une aire géographique prend contour. Les anthroponymes véhiculent à titre connotatif des indications sur l'appartenance géographique de leur porteur, et fonctionnent à ce titre comme fixateur de cadre géographique. On peut d'ailleurs noter, à l'instar du fonctionnement des didascalies que les localisations dans le temps et dans l'espace fonctionnent de façon comparable, comme on le voit en (3) et dans l'exemple suivant:

(4) Helena / Menelaos / Hermione / Paris (Hildesheimer; Das Opfer Helena).

La variété des localisations est immense:

(5) Satan / Lilith / Beelzbuth / Astaroth / Asmodi etc. (Mehring; Die höllische Komödie).

Ceci peut d'ailleurs être la source d'effets comiques. Quand on lit, dans la liste de la même pièce:

(6) Und auf Erden:

Cesare,

Plutus / Junius / Audax: Deutsche Reporter im Völkerbund (Mehring; Die höllische Komödie).

Le lecteur est pris à contre-pied: l'attente suscitée par les anthroponymes ne correspond guère à leur affectation diégétique, car il n'est pas peu surprenant de lire que Plutus, Junius et Audax sont des désignateurs de reporters allemands auprès la Société des Nations!

Ce type de signification ne devrait pas poser trop de problèmes en matière de traduction, même si des difficultés peuvent toutefois surgir lors des conventions de traduction d'une langue à l'autre d'une tierce langue, comme le montrent les exemples (4) et (7):

\begin{tabular}{|l|l|}
\hline Texte allemand & Texte français \\
\hline Wang & Wang \\
\hline Shen Te & Shen Té \\
\hline Shui Ta & Shui Ta \\
\hline Die Hausbesitzerin Mi Tzü & Madame Mi Tsu, propriétaire. \\
\hline (Brecht; Der gute Mensch von Sezuan) & La bonne âme de Se Tchouan \\
\hline
\end{tabular}

On le voit, des modifications apparaissent, de l'ordre de la correspondance graphie / phonie pour cet exemple. Sans doute s'agit-il d'une sorte de convention telle que celle qui poussait par exemple G. de Nerval dans sa traduction du Faust de Goethe 
à traduire Wagner par Vagner, ce qui ne laisse pas de faire surgir des questions relatives à deux autres types de connotations énonciatives tels qu'ils seront présentés ci-dessous.

Il faut encore noter que ces connotations permettent d'ancrer l'ensemble de la diégèse de niveau 2, mais aussi parfois de ne situer qu'un ou quelques personnages, avec effet contrastif vis-à-vis des autres:

(8) Kokol / Polpich / Curucu / Rossignol (Weiss; Die Verfolgung und Ermordung Jean Paul Marats...);

(9) Harras / Lüttjohann / Korrianke / ... / Buddy Lawrence (ein amerikanischer Journalist) (Zuckmayer; Des Teufels General).

Après la liste des personnages de haute stature, clairement connotés français, on trouve dans la liste de la pièce de $\mathrm{P}$. Weiss le nom de ces quatre autres personnages (en 8), et l'on voit bien tout l'effet comique (en tout cas en fort contrepoint) signifié par ces anthroponymes.

De la même façon, en 9, le nom signifie bien aussi la provenance du personnage diégétique américain.

Il faut pareillement ajouter que les localisations sont aussi, le cas échéant, intergermaniques dans la mesure où elles concernent des différenciations, par exemple entre la Prusse (Schmarowski et Wulkow dans une pièce de Hauptmann), et l'Autriche (Frau Haberl dans une pièce de F. X. Kroetz) etc. Que l'on songe dans le théâtre français à Escartefigue ou Panisse!

Enfin, certains choix se révèlent tout à fait significatifs quand les noms propres se caractérisent tous par une absence de forte connotation sur ce plan. Chez F. X. Kroetz, certaines listes sont remarquables de ce point de vue:

(10) Franz Schritt / Maria Schritt / Lorenz / Damian (Kroetz; Bauerntheater);

(11) Helga / Emmi / Hermann / Edgar / etc. (Kroetz; Nicht Fisch Nicht Fleisch).

La signification globale est ici patente, concernant le manque de distinction particulière, le fait qu'aucun système de repérage spécifique n'est mis à la disposition du lecteur. Mais sans doute l'analyse de tels phénomènes relève-t-elle davantage de l'étude littéraire dramaturgique.

Certains cas créent de ce point de vue une sorte d'embarras, qui découle justement de la difficulté liée à l'absence de connotation situative:

(12) Sakko / Oi (Brasch; Mercedes)

Je ne résiste pas au plaisir de citer S. Beckett, qui avec ses quatre prénoms centrifuges de Fin de partie bloque tout enracinement dans un hic et nunc géographique quelconque: Nagg, Nell, Hamm, Clov.

\subsection{Connotation énonciative $=$ situative diégétique: localisation sociologique}

Le caractère situatif des anthroponymes par le biais de leur signification concerne aussi les sphères sociologiques, et des charges affectives. Point n'est besoin de détailler ici beaucoup

(13) von Wehrhahn $\neq$ Fielitz (Hauptmann; Der rote Hahn).

Certains cas se révèlent cependant intéressants, du point de vue de la traduction notamment. 
Dans la pièce de F. Dürrenmatt, Der Besuch der alten Dame, un personnage, Claire Zachanassian, est appelé de plusieurs façons, et la traduction correspond à peu près (mis à part peut-être une connotation géographique perdue dans le surnom Kläri):

\begin{tabular}{|l|l|}
\hline Claire Zachanassian & Claire Zahanassian \\
\hline Kläri & Clairette \\
\hline Die Kläri Wäscher & Clara Wäscher \\
\hline
\end{tabular}

En revanche, dans le Faust de Goethe, certaines nuances portées par le nom du personnage féminin ne sont guère traduites:

\begin{tabular}{|l|l|}
\hline Margarete & Marguerite \\
\hline Gretchen & Marguerite \\
\hline
\end{tabular}

La signification des deux formes en allemand n'est cependant pas vraiment équivalente, à tout le moins sur le plan connotatif / affectif.

\subsection{Connotation énonciative $=$ situation discursive et renvoi intertextuel}

Un dernier type de signification d'ordre énonciatif doit enfin être noté: il s'agit de la fonction remplie par certains anthroponymes de marqueurs intertextuels par le biais de ce type de connotation: Oberon et Titania, présents dans le Faust de Goethe, ne peuvent s'interpréter qu'en référence à Shakespeare (Le songe d'une nuit d'été).

Cette épaisseur intertextuelle est également bien présente lorsque l'on lit la liste des personnages de la pièce de P. Handke:

(16) Ein Mauerschauer / ein Spielverderber / Ein junger Schauspieler / Eine junge Schauspielerin / Ein altes Paar / parzival / ... (Handke; Die Kunst des Fragens).

Le problème de traduction ne se pose alors qu'en relation avec les connaissances culturelles ou la pénétration de la référence intertextuelle. Pour Parzival, il ne devrait y avoir aucun problème, mais les choses ne sont peut-être pas aussi simples: doit-on choisir Perceval ou Parsifal? La différence de signification n'est pas peu importante!

Un dernier cas mérite d'être noté: les noms / prénoms, qui à l'instar des noms dans les pièces de Molière connotent précisément le discours théâtral qu'ils contribuent ainsi à constituer: Argan, Orgon, Oronte, Bélise, Éraste, etc. ${ }^{4}$

\subsection{Connotation métalinguistique: l'aura du dénoté}

Dernier registre des connotations, plus original, et présentant de bien grandes difficultés sur le plan de la traduction, je veux présenter ce type de connotation spécifique aux noms propres, et qui correspond au dénoté du signe lexical (simple ou complexe) qui donne son signifiant au nom propre.

Ceci nous ramène au cas de Wagner ci-dessus, et à la traduction désastreuse en Vagner qui bloque en tout état de cause toute dénotation; on peut certes objecter que le signe /Wagner ${ }^{5}$ / est devenu tellement diaphane que le signifié commun a disparu. Mais certains exemples montrent que l'on ne peut l'évacuer si facilement. 
Peut-on ainsi penser que le personnage de Goethe s'appelle Frosch ${ }^{6}$ impunément, alors qu'il est traduit dans le texte français par Frosch!

Ce n'est pas en vain qu'un personnage d'une pièce d'A. Schnitzler, professeur des maladies de la gorge, s'appelle Dr. Schreimann ${ }^{7}$ !

Pas besoin de multiplier les exemples. Le théâtre français regorge ainsi d'exemples comparables: Me de Tubéreuse ${ }^{8}$, la châtelaine de la pièce d'Obaldia Genousie).

Cette disposition sémantique pose parfois des problèmes, lorsque la frontière n'est plus nette entre nom propre à part entière, même s'il contient un dénoté commun à titre connotatif, et cette même description commune:

\section{(17) Mann / Frau / Zwei Kinder / Gast / Warner (Weiss; Nacht mit Gästen)}

Le terme Warner est ici ambigu: est-ce un nom propre ou la désignation d'un personnage par la nomination de sa fonction, celle d'avertir? Nul doute que la traduction va ici poser certaines difficultés.

Pour terminer je désire présenter une façon à mon sens évidente de faire jaillir des connotations, à partir de vocables non existants auparavant, mais dans un autre registre que le seul discours théâtral ${ }^{9}$. Il s'agit de la façon dont G. Bourdet baptise ses personnages, le héros éponyme (Bourdet; Le Saperleau) et les deux héroïnes: Apostasie et Morvianne. Un simple jeu d'associations paradigmatiques fait surgir de bien réels signifiés de connotation.

\section{Les limites du système}

Ces derniers exemples montrent bien que la limite entre noms propres et noms communs n'est peut-être pas aussi nette qu'on pouvait le penser avant une telle étude, du moins dans le domaine théâtral.

Car ce type de discours favorise cette sorte de rapprochement. D'une part en effet, formellement, par leur situation à gauche dans la liste, certains éléments gardent à l'intérieur du discours théâtral la fonction de désignation rigide, alors qu'ils sont clairement des noms communs, comme en (17) ou (19).

Mais certains cas sont encore plus indécidables; ils résultent de la tension à l'extrême du système, où le signifiant ne permet plus guère de se prononcer:

(19) Personen: Der Mann $\cdot$ Die Frau $\cdot K \cdot M \cdot$ Zweiter Mann $\cdot$ Kellner / Chef (Strauß; Kalldewey, Farce)

Le dernier cas est représenté par la pièce de P. Weiss (Gesang vom Lusitanischen Popanz) où, mis à part le fantoche, désigné comme tel, les autres personnages ne portent pour tout nom propre qu'un chiffre!

\section{Conclusion}

Grâce à ce dernier exemple, il est possible de revenir sur la question posée à propos du sens des noms propres. Il apparaît qu'outre la signification non lexicale, procédurale, les anthroponymes portent aussi donc un sens lexical bien réel, même s'il se limite au registre du connotatif. Et c'est sans doute la raison pour laquelle certains auteurs ont précisément recours à certaines appellations (chiffres, initiales) qui, sous cette forme, sont seules susceptibles de ne pas véhiculer un sens qui leur paraît pour une raison ou une autre comme une sorte de parasitage. 
Et de ce fait, la barrière entre noms propres et noms communs semble s'être assez considérablement réduite, du moins si l'on en juge par les phénomènes tels qu'ils transparaissent dans les textes de théâtre.

\section{NOTES}

1. Certains personnages diégétiques n'ont cependant pas toujours droit à l'honneur de figurer dans cette liste. Sur ce point cf. (Gallèpe 1997b et 1997c).

2. Les termes connotation $\neq$ dénotation sont ici pris dans une perspective logique.

3. Traits sémantiques distinctifs, permettant d'identifier une classe d'objets.

4. Je ne peux ici évoquer la signifiance des noms propres telle qu'elle a pu être analysée par M. Arrivé lors du colloque organisé à Chambéry en novembre 2002 "Littérature et linguistique: diachronie / synchronie - Autour des travaux de Michèle Perret», à propos des noms propres du théâtre de Molière. Je renvoie entre autres à la façon dont le prénom «George» signifie, dans la structure de son signe, la scission du porteur par l'or: ge/or/ge.

5. Wagner signifie en allemand «charron».

6. Frosch signifie en allemand «grenouille».

7. En allemand, cet anthroponyme est constitué de deux signes signifiant respectivement «cri» et «homme».

8. Il convient de remarquer ici l'épaisse stratification de la signification: le dénoté du nom commun «tubéreuse» est connoté dans le nom propre, mais celui-ci a également comme sème afférent le trait /très parfumé/, qui ne peut pas ne pas rejaillir, par métonymie, sur le porteur de ce nom. Par ailleurs, le signifiant en «-euse» peut lui aussi être porteur de signifié de connotation plutôt négative (// vénéneuse, véreuse, etc.).

9. Le monde de l'industrie est ainsi grand consommateur de nouveaux noms propres pour désigner ses produits. Les choix dépendent également des réseaux connotatifs que ces appellations actualisent chez les locuteurs des différents pays visés par la commercialisation de ces produits.

\section{RÉFÉRENCES}

Gallèpe, T. (1997a): Didascalies. Les mots de la mise en scène, Paris, L'Harmattan.

GALLÈPE, T. (1997b): «La mise en place de l'interaction par les didascalies dans der Hofmeister et die Soldaten de J. M. R. Lenz», Les Nouveaux Cahiers d'Allemand No spécial concours, p. 111126.

Gallèpe, T. (1997c): «Le jeu des didascalies sans hasard», Champs du Signe, № Spécial concours, Toulouse, Presses Universitaire du Mirail, p. 71-88.

Kerbrat-Orecchioni, C. (1977): La connotation, Lyon, Presses Universitaires de Lyon.

Rastier, F. (1996): Sémantique interprétative, Paris, PUF.

\section{SOURCES}

Beckett, S. (1957): Fin de partie, Paris, Éd. de Minuit (1957).

Bourdet, G. (1982): Le Saperleau, Paris, Solin (1982).

Brasch, T. (1983): Mercedes, in Rach, R. (ed.) Theater Heute, Frankfurt am Main, Suhrkamp Verlag (1985).

Brecht, B. (1943): Der gute Mensch von Sezuan, Frankfurt am Main, Suhrkamp Verlag (1973).

BRecht, B. (1959): Die heilige Johanna der Schlachthöfe, Berlin, Suhrkamp Verlag (1971).

Büchner, G. (1835): Dantons Tod in Werke und Briefe, München, DTV - Gesamtausgabe (1971).

Obaldia, R. de (1960): Genousie, Paris, Grasset (1966).

Dürrenmatt, F. (1956): Der Besuch der alten Dame, Zürich, Die Arche (1956).

Goethe, J. W. (1829): Faust. Reclam, Stuttgart, Universal Bibliothek (1972).

Handke, P. (1989): Die Kunst des Fragens, Frankfurt am Main, Suhrkamp Verlag (1994).

Hauptmann, G. (1901) : Der rote Hahn, Frankfurt am Main, Ullstein - Theater Texte (1979).

Hildesheimer, W. (1969): Das Opfer Helena in Zwei Komödien (1969), Frankfurt am Main, Fischer Bücherei. 
Носннитн, R. (1963): Der Stellvertreter, Hamburg, Rowohlt Taschenbuch Verlag (1967).

Hochнuth, R. (1974): Inselkomödie, Hamburg, Rowohlt Taschenbuch Verlag (1973).

Kroetz, F. X. (1983): Nicht Fisch Nicht Fleisch in Rach, R. (ed.), Theater Heute, Frankfurt am Main, Suhrkamp Verlag (1885): Frankfurt am Main.

Kroetz, F. X. (1991): Bauerntheater, Frankfurt am Main, Suhrkamp Verlag (1991).

Menring, W. (1933): Die höllische Komödie in Die höllische Komödie - drei Dramen, Frankfurt am Main, Ullstein - Werkausgaben (1981).

Schnitzler, A. (1912): Professor Bernhardi in Professor Bernhardi und andere Dramen, Frankfurt am Main, Fischer Taschenburg Verlag (1979).

Strauss, B. (1981): Kalldewey, Farce in Rach, R. (ed.), Theater Heute, Frankfurt am Main, Suhrkamp Verlag (1985).

Weiss, P. (1963): Nacht mit Gästen in Gesang vom Lusitanischen Popanz und andere Stücke, München, DTV - Sonderreihe 78 (1969).

Weiss, P. (1964): Die Verfolgung und Ermordung Jean Paul Marats dargestellt durch die Schauspielergruppe des Hospizes zu Charenton unter Anleitung des Herrn de Sade, Frankfurt am Main, Suhrkamp Verlag (1968).

Weiss, P. (1967): Gesang vom Lusitanischen Popanz in Gesang vom Lusitanischen Popanz und andere Stücke, München, DTV - Sonderreihe 78 (1969).

Zuckmayer, C. (1931): Der Hauptmann von Köpenick, Frankfurt am Main, Fischer Taschenbuch Verlag (1976).

Zuckmayer, C. (1945): Des Teufels General, Fischer Taschenbuch Verlag (1973). 